\title{
6. Internationaler CAMLOG Kongress
}

Unter dem Motto „Tackling everyday challenges“ fand der diesjährige 6. Internationale CAMLOG Kongress vom 9.-11. Juni in Krakau statt. Mehr als 1300 Teilnehmer aus der ganzen Welt und 76 internationale Referenten und Moderatoren waren zu diesem herausragenden Event angereist. Krakau und das ICE Kongresszentrum erwiesen sich als perfekte Wahl für diesen Kongress, der erfolgreich Forschung und Praxis verband und als zentrale Themen die Gewebestabilität, die Behandlungsplanung sowie die digitale Zahnheilkunde thematisierte

Wie gelingen langzeitstabile, ästhetische Implantatversorgungen mit guter Voraussagbarkeit? Was ist bei der Hardware zu beachten, was bei chirurgischen und prothetischen Methoden? Welche Rolle spielen Diagnostik und Planung? Wie können periimplantäre Hart- und Weichgewebe stabil gehalten werden? Zu diesen Fragen und mehr gaben erfahrene sowie auch junge Kliniker überzeugende Antworten zu den Themen: Vertikale Position vor Verbindungstyp, Weichgewebe schützt Knochen, Abutments mit Attachment, iSy - Patienten gewinnen mit neuem Konzept, Kurze Implantate versus Augmentation, Ist Periimplantitis vermeidbar?

\section{The Battle - kontroverse Themen heiß diskutiert}

Den Höhepunkt der beiden Kongresstage bildete ebenfalls den Abschluss. In „Streitgesprächen“ wurden unterschiedliche Meinungen heiß diskutiert. Das Publikum hatte jederzeit die Möglichkeit Fragen via APP einzubringen, welche dann in der Diskussionsrunde aufgegriffen wurden. So zeigte Privatdozent Dr. Markus Schlee (Forchheim) Zweifel an der ätiologischen Hauptrolle des Biofilms. Auch andere Referenten bewerteten dies in ihren Beiträgen ähnlich. Oft sei unklar, warum manche Patienten Periimplantitis bekommen, andere dagegen nicht. Da verfügbare Therapiemethoden eine unsichere Prognose hätten, bevorzugt Schlee in vielen Fällen Explantationen und gegebenenfalls erneute Implantationen. Professor Frank Schwarz (Universität Düsseldorf) hielt dem entgegen, dass noch in keiner Studie ein Knochenabbau ohne biofilminduzierte Entzündung nachgewiesen werden konnte. Auch therapeutisch seien verschiedene Methoden nachweisbar erfolgreich, je nach Situation müssten Implantatoberflächen mit rotierenden Instrumenten geglättet werden. Alle Referenten waren sich einig, dass eine ganze Reihe von Faktoren periimplantäre Entzündungen begünstigen, darunter Parodontitis, Qualität und Quantität von Weichgeweben, Implantatposition, Zementreste im Sulkus (Gegenmittel: Faden legen) und Oberflächenbeschaffenheit von Implantat, Aufbau und Restauration. Weniger gut ist die ätiologische Rolle von Wirtsfaktoren erforscht, wie systemische Erkrankungen, Immunologie und genetische Disposition. Im Zweifel sollte, zum Beispiel nach Einschätzung von Professor Myron Nevins, auf Implantate verzichtet und nach Möglichkeit eine Brücke eingegliedert werden.

\section{Digital Dentistry Vor-Kongress}

Erstmalig hat im Vorfeld des Internationalen CAMLOG Kongresses ein Vor-Kongress, welcher ganz dem Thema der Digitalen Zahnheilkunde gewidmet war, stattgefunden. Implantation und Prothetik lassen sich am Computer in neuer Dimension planen; standardisierte Methoden und Materialqualität erlauben besser voraussagbare Ergebnisse. Dies funktioniert aber nur mit geeigneter Technologie und fundiertem Wissen wie Experten aus Hochschule, Praxis und Labor in Krakau zeigten.

\section{Young Generation}

Eines der Ziele der CAMLOG Foundation ist es, den wissenschaftlichen Nachwuchs zu fördern. Dies erfolgt einerseits durch die Ausschreibung des Research Awards und die Möglichkeit der Teilnahme am Posterwettbewerb, bei welchem in diesem Jahr mehr als 60 Poster aus 9 Ländern konkurrierten. Als Neuheit hatten die Posterteilnehmer die Chance, ihr Poster mündlich zu präsentieren. Der gut besuchte Speakers Corner generierte reges Interesse und während einige Referenten bereits erprobte Redner waren, bot sich anderen die erstmalige Gelegenheit zur Präsentation.

\section{Fazit}

Der 6. Internationale CAMLOG Kongress bot einmal mehr eine gelungene Mischung aus hochstehender Wissenschaft, relevantem Praxisbezug und einer mitreißenden Stimmung. Der Krakauer Kongress brachte zu aktuellen Fragen eine Fülle von Informationen, die sich direkt für die tägliche Praxis nutzen lassen. Faszinierend war auch der Austausch zwischen Experten, von denen viele sowohl in eigener Praxis als auch in der Forschung erfolgreich sind.

\section{Gi/ZWR}

Nach einer Pressemitteilung der CAMLOG Foundation, CH - Basel
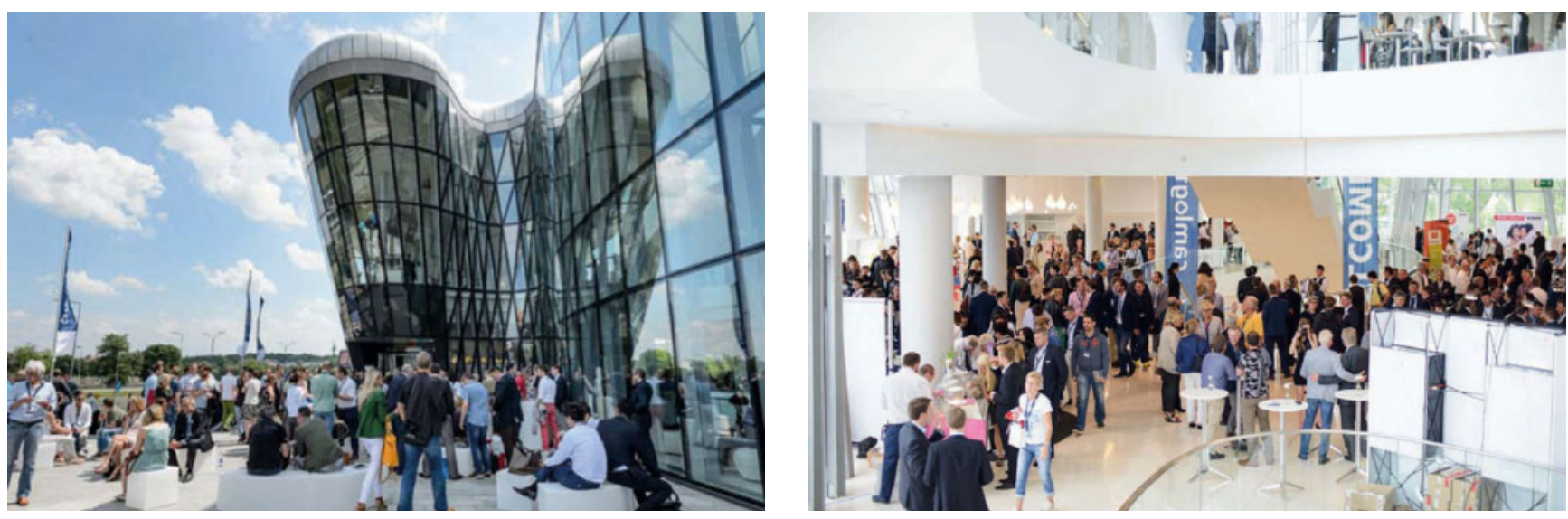